\title{
Problemática medioambiental y participación ciudadana: una aplicación al caso de los residuos urbanos
}

\section{Berné, C. Garcés, A. Lafuente, M. Pedraja y P. Rivera **}

\section{Introducción}

El análisis de la evolución de la regulación medioambiental en España debería remontarse al inicio de la década de los sesenta, momento en el que surgen los primeros vestigios relativos a normativa medioambiental ${ }^{1}$. No obstante, es en los comienzos de los años setenta cuando las repercusiones políticas y económicas de los asuntos relacionados con la protección del medio ambiente comienzan a manifestarse de forma notable en nuestro país. Así, en 1972, año en el que se celebró la Conferencia de Naciones Unidas sobre el Medio Humano, se creó en España la Comisión Interministerial del Medio Ambiente (CIMA) para coordinar las actuaciones públicas en materia medioambiental. En 1986, la adhesión española a lo que hoy en día constituye la Unión Europea señaló la consolidación de las políticas públicas, a través de los sucesivos Programas de Acción en Materia de Medio Ambiente. Así, se han venido estableciendo exigencias medioambientales que condicionan la actividad de los principales agentes implicados: las empresas, las administraciones y los ciudadanos.

Aunque tradicionalmente la gestión de las exigencias medioambientales se ha situado en el ámbito público, la dimensión del problema ha determinado una nueva formulación de la protección medioambiental en favor de una mayor coordinación entre agentes públicos y privados. Es así como las empresas deben enfrentarse a nuevas restricciones medioambientales que son canalizadas fundamentalmente a través del proceso político-legal desarrollado por las autoridades competentes y a través de las exigencias del mercado. Como resultado de estas restricciones y de la presión ejercida por los medios de comunicación, las empresas modifican sus procesos productivos, replantean el diseño y componentes de sus productos, orientan a los ciudadanos hacia el adecuado uso y desecho de los mismos y fomentan el reciclado y la reutilización de los subproductos y residuos.

Las administraciones, por su parte, deben crear un marco industrial, social, económico y regulador para que, tanto las empresas como los ciudadanos, dispongan de la información $y$ de los medios pertinentes para prevenir y tratar los problemas medioambientales. En lo que se refiere a la infraestructura industrial, las administraciones no sólo deben proporcionar unos adecuados servicios de recogida y depósito de residuos y subproductos, sino que están obligadas a promover también una oferta medioambiental tecnológica (maquinaria y equipos limpios) y de servicios (consultoras, asesorías, seguros...). Desde el punto de vista social, deben promover la cultura y la concienciación de los ciudadanos y las empresas, a través de adecuadas campañas de información y educación medioambiental. En lo referente a la infraestructura económica, las administraciones están llamadas a establecer políticas económicas ecológicamente sostenibles, y que proporcionen señales adecuadas para dirigir a las empresas a la sostenibilidad, tales como la concesión de subvenciones o de ventajas fiscales a aquellas que inviertan en proyectos de menor agresividad ambiental. Además, las administraciones deben establecer una regulación en 
materia de medio ambiente, así como los medios de supervisión y control necesarios para su efectivo cumplimiento.

Sin embargo, las tendencias sociales de respeto al medio ambiente no podrían traducirse en la obtención de resultados positivos sin la participación activa de los ciudadanos. Los ciudadanos, como miembros de una comunidad, deben manifestar su apoyo a las empresas en las que trabajan y a las administraciones que gobiernan sus comunidades, en la adopción de medidas en favor del medio ambiente, a través de los cambios que sean necesarios en sus costumbres y estilos de vida. En este sentido, el V Programa Comunitario de Política y Actuación en Materia de Medio Ambiente y Desarrollo Sostenible ${ }^{2}$, a diferencia de los anteriores, menciona expresamente la responsabilidad social de ciudadanos y consumidores para la consecución de un equilibrio entre los beneficios privados a corto plazo y los beneficios a largo plazo para la sociedad.

Pese a la gran notoriedad de las tecnologías para la resolución de los problemas medioambientales, existe una escasa investigación en nuestro país en lo que respecta a las exigencias que se le plantean al ciudadano y a las actividades que éste puede desarrollar para implicarse en la defensa del medio ambiente. Es aquí donde encuentra su objetivo este trabajo, que pretende analizar determinadas percepciones, actitudes y comportamientos de los ciudadanos, que implican compromiso personal con el medio ambiente.

La tesis implícita en este trabajo indica que, en la resolución de los problemas medioambientales, existe una restricción de tipo social que, en bastantes casos, implica adoptar soluciones técnicas menos eficaces y/o económicamente más costosas. La dimensión social inherente a la resolución de los problemas medioambientales resulta especialmente relevante cuando la elección de una tecnología concreta para la resolución de un problema medioambiental depende de manera fundamental de la actitud, de aceptación o rechazo, de la sociedad. Podemos pensar, por ejemplo, en los depósitos de seguridad para el almacenamiento geológico profundo de residuos tóxicos y peligrosos. La eficacia técnica de dicha solución tecnológica ha sido adecuadamente demostrada y, en general, la sostenibilidad económica no constituye una barrera relevante para su adopción. Sin embargo, la dificultad práctica de obtener la aceptación de una comunidad para la ubicación del depósito en sus dependencias sí constituye una barrera relevante.

En otras ocasiones, la dimensión social adquiere relevancia en el sentido de que la eficacia de una determinada tecnología depende del comportamiento, activo o pasivo, de los individuos. Es bien conocido, por ejemplo, que la utilización de medios de transporte públicos por parte de los ciudadanos constituye una fuente potencial de resolución a la acumulación atmosférica del principal gas de efecto invernadero, el $\mathrm{CO}_{2}$. De nuevo, la eficacia de esta solución depende, no tanto de su validez tecnológica o de su sostenibilidad económica como de la consistencia de los comportamientos sociales.

Ejemplos como los propuestos justifican el interés por conocer cómo actúan los individuos implicados en la puesta en práctica de soluciones a los problemas de tipo medioambiental, cuáles son sus características socio-demográficas y qué actitudes y comportamientos manifiestan. Para ello, nada mejor que examinar un caso, la gestión de los residuos urbanos a través de la clasificación doméstica y su depósito en contenedores establecidos a tal efecto en los núcleos urbanos. La base de datos que sirve al objeto de la investigación la proporciona una encuesta, realizada en la ciudad de Zaragoza durante los meses de abril y mayo de 1999, referida a la participación ciudadana en el actual Programa de Recogida Selectiva de Residuos Urbanos.

El trabajo presentado se estructura en varias partes. En primer lugar, se plantea el problema medioambiental generado por los residuos urbanos. Posteriormente, tras la presentación de la información recogida a través de una encuesta, se muestran los principales resultados obtenidos, así como las conclusiones más relevantes.

\section{Los residuos urbanos (RU)}

El nivel de desarrollo alcanzado por las sociedades occidentales da lugar a la generación de cantidades ingentes de toda clase de residuos, incluidos entre ellos los residuos urbanos. Según la Ley de Residuos 10/1998, de 21 de abril, se consideran residuos urbanos:

- Los generados en los domicilios particulares, comercios, oficinas y servicios, así como todos aquellos que no tengan la calificación de peligrosos y que, por su naturaleza o composición, puedan asimilarse a los producidos en los anteriores lugares o actividades.

- Los procedentes de la limpieza de vías públicas, zonas verdes, áreas recreativas y playas.

- Los animales domésticos muertos, así como muebles, enseres y vehículos abandonados.

- Los residuos y escombros procedentes de obras menores de construcción y reparación domiciliaria.

En España se generaron, en 1996, en torno a 15 millones de toneladas de este tipo de residuos, lo que supone una producción de $388 \mathrm{Kg}$ por habitante y año. La distribución territorial de estos residuos ${ }^{3}$ muestra que la mayor producción por habitante procede de las Comunidades Autónomas de Baleares, Madrid, Canarias y Cataluña. Teniendo en cuenta las diferencias en la densidad de población entre comunidades, Cataluña, 
Madrid y Andalucía concentran casi el 50\% del total de los residuos urbanos generados en nuestro país.

La composición media de estos residuos muestra que, en 1998, la fracción correspondiente a materia orgánica representa, en peso, el $50 \%$ del total, estando el $50 \%$ restante constituido en su mayor parte por residuos de envases y embalajes, $31 \%$; el resto de tal porcentaje se corresponde con papel impreso, textil, goma y caucho, artículos plásticos, metales no procedentes de envases y residuos especiales ${ }^{4}$.

El vertido incontrolado de los residuos urbanos perjudica al medio ambiente a través de la contaminación del suelo, agua y aire; da lugar a la proliferación de especies antropófilas que perjudican a la salud; deteriora los ecosistemas naturales amenazando la biodiversidad y, además, constituye un notorio despilfarro de materias primas.

Hasta hace poco tiempo, las soluciones desarrolladas para evitar estas negativas consecuencias se han orientado casi exclusivamente hacia el sistema clásico de depósito en vertederos controlados. Hoy en día, sin embargo, se han empezado a considerar opciones tecnológicas adicionales para la gestión de los residuos urbanos. En términos generales, estas opciones pretenden alcanzar objetivos de recuperación y reciclado de una parte importante de los residuos generados, y han sido recogidas en todas las políticas de actuación en materia de medio ambiente de los países más avanzados del mundo y, por supuesto, en la Unión Europea, dentro de su V Programa de Acción en Materia de Medio Ambiente 5 .

Así, la Directiva 94/62/CEE, relativa a envases y residuos de envases, establece como principios básicos la recuperación y la minimización. Esta Directiva ha sido traspuesta al Derecho interno español a través de la promulgación de la Ley 11/1997, de 24 de abril, de Envases y Residuos de Envases, que establece unos objetivos de reciclado y valorización de los residuos de envases y embalajes que deben cumplirse en todo el territorio nacional a partir de junio del año $2001^{6}$. El ámbito de aplicación de esta Ley son todos los envases y residuos de envases puestos en el mercado y generados en el territorio nacional con independencia de su carácter doméstico, comercial o industrial.

La obligatoria aplicación de esta Ley, en lo que respecta a los envases de nuestro interés (domésticos), junto con el crecimiento de los núcleos urbanos, ha motivado que los costes relacionados con el tratamiento de los residuos urbanos supongan una proporción creciente de los presupuestos municipales ${ }^{7}$. Los costes de estas operaciones, para los municipios implicados, pueden ser menores si los ciudadanos se involucran en el proceso de gestión de los residuos que generan, clasificándolos por fracciones y depositándolos en contenedores específicos (Franco y HuerTa, 1996).

En España existen algunas experiencias notables. Por ejemplo, la llevada a cabo en la Comarca de Pamplona, donde el
$75 \%$ de la población procede diariamente a la separación doméstica de basuras. Esto ha hecho posible que, en dicha comarca, se reciclara entre enero y agosto de 1994 el 38,67\% de los residuos reciclables, superándose el objetivo marcado por la Unión Europea para el año 2001 situado en el logro de un $25 \%$ de reciclado.

Para que el sistema de recogida selectiva cumpla sus objetivos, los ciudadanos deben participar en el mismo llevando a los contenedores los materiales específicos. Éste es el tema de nuestro interés, analizar las características, actitudes y creencias de los individuos que están participando en los sistemas de recogida establecidos, así como su forma real de actuación.

\section{Método}

El análisis del caso propuesto tiene como objetivo analizar las percepciones, actitudes y comportamientos relacionados con el desarrollo del Plan de Ordenación de la Gestión de Residuos Urbanos de la Comunidad Autónoma de Aragón.

La literatura sobre gestión de problemas medioambientales se ha ocupado relativamente poco de los comportamientos sociales, es decir, de los procesos generadores de valoración de problemas y soluciones, y de los procesos conformadores de actitudes, favorables o desfavorables, respecto de iniciativas orientadas a la solución de problemas. Así, la mayor parte de los estudios ha puesto en relación la sensibilidad genérica respecto del medio ambiente con características socio-demográficas, aunque debe señalarse que, más recientemente, se ha pretendido dar cuenta de las actitudes respecto de problemas y soluciones específicos como el reciclado de residuos urbanos o el ahorro de energía. Así, dentro de la literatura especializada que ha estudiado los factores que condicionan la mayor o menor participación ciudadana en procesos de reciclaje, los primeros estudios ponen de manifiesto el hecho de que existe una mayor preocupación por estos temas entre gente joven y con alto nivel de educación (ToGNACCI et al., 1972; McEvoY, 1972; BuTTEL y FLINN, 1974, 1978) y entre los residentes de áreas urbanas (TREMBLAY y DunlaP, 1978; LOWE y PinHeY, 1982). DunlaP y CaTton (1979) encontraron como factores determinantes la edad, la residencia, la educación y la ideología política, no existiendo relación con el nivel de renta; VINING y EBREO (1990) detectaron, sin embargo, relación con edad y renta. Igualmente, la distancia entre la residencia y el lugar de depósito de los residuos explica también en buena parte el grado de participación ciudadana (Finnie, 1973; Witmer y Geller, 1976; Jacobs, Balley y Crews, 1984; FRANCO Y HUERTA, 1996).

Así pues, la explicación y, en su caso, la predicción de los comportamientos sociales respecto al medio ambiente 


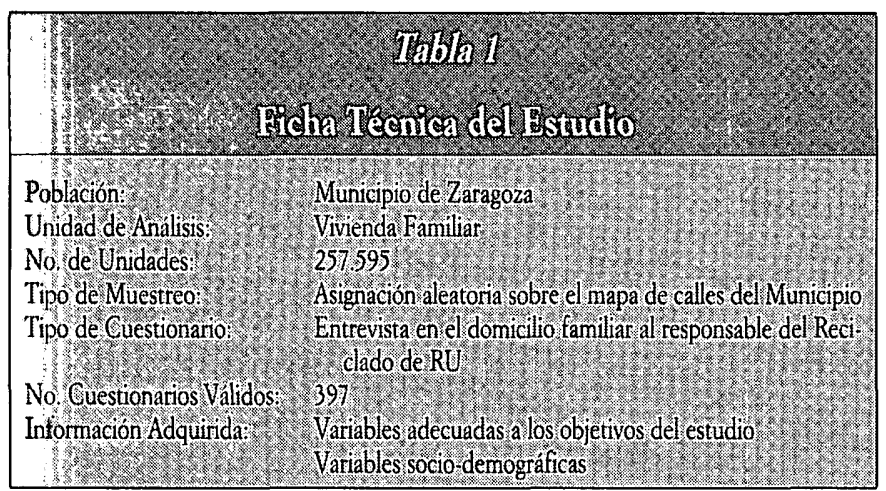

debe considerar diferentes tipos de variables entre las que destacan las características personales, el conocimiento previo del problema, las actitudes generales y específicas, las valoraciones de la iniciativa medioambiental y las dificultades/costes percibidas para la adhesión a la misma. Es precisamente esto lo que nos proponemos a través del examen de la recogida selectiva de basuras como elemento de gestión de los residuos urbanos.

\section{Base de Datos}

El trabajo realizado se inicia con la elaboración de un cuestionario y la realización de una encuesta en la ciudad de Zaragoza, durante los meses de abril y mayo de 1999 (ver Tabla 1). La información obtenida se refiere a la participación de los hogares encuestados en los programas vigentes de recogida selectiva de residuos urbanos, conocimiento de los mismos, percepciones sobre diferentes problemas medioambientales, actitudes frente a las políticas públicas en la materia, dificultades percibidas y características socio-demográficas. A excepción de las características socio-demográficas, toda la información recogida ha sido medida a través de escalas de cinco puntos.

Las características socio-demográficas de los encuestados, reflejadas en la Tabla 2, muestran la presencia de un 32\% de hombres, una edad comprendida fundamentalmente entre los dieciocho y los cuarenta y nueve años, niveles de estudio de bachiller y superior mayoritariamente, unidades familiares compuestas, en término medio, por cuatro miembros y con unos ingresos anuales comprendidos entre 1 y 4 millones de pesetas mayoritariamente.

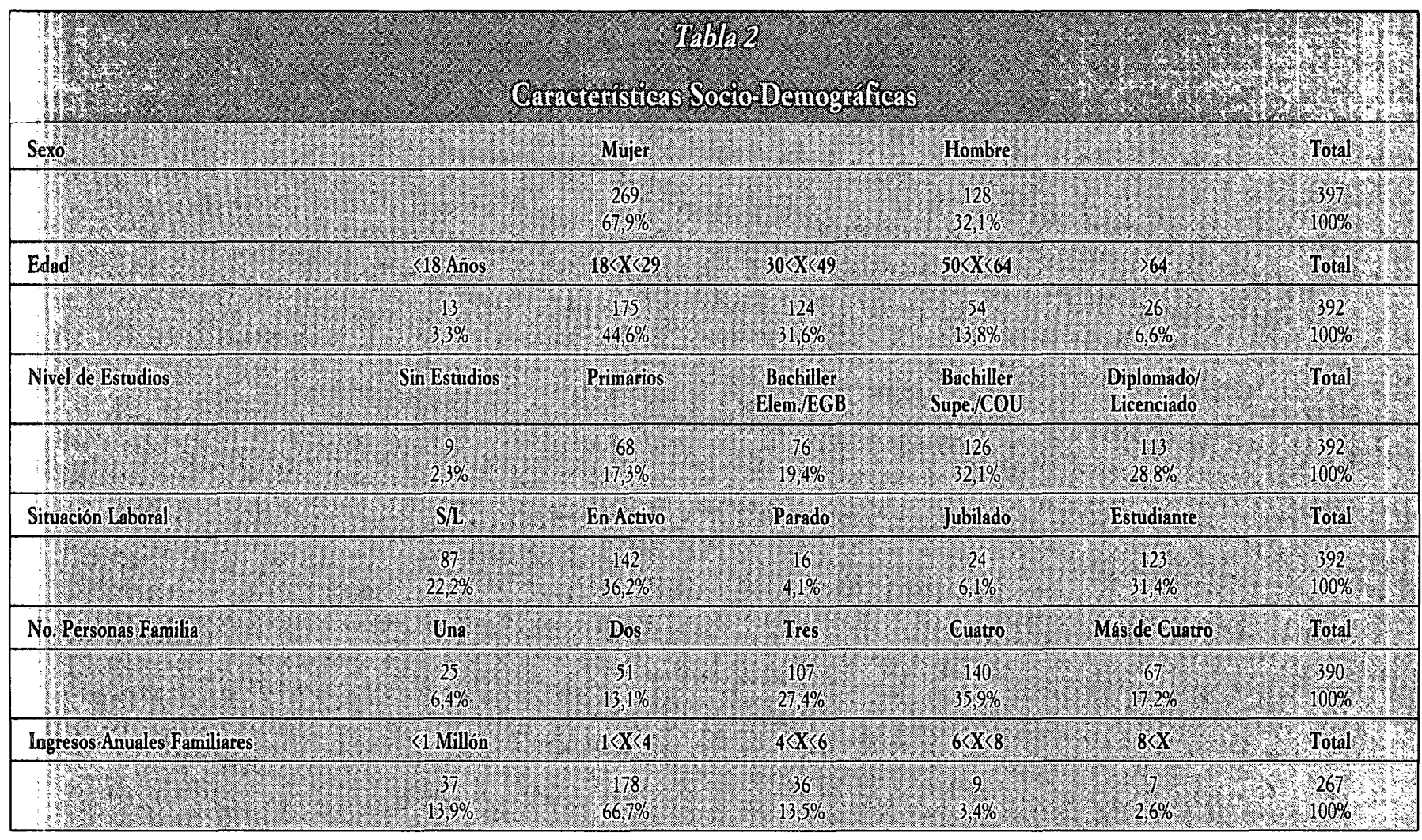




\section{Análisis y resultados}

La primera cuestión de interés para el análisis es la relativa al nivel de conocimiento-percepción manifestado por los encuestados sobre los problemas medioambientales en general y sobre el problema derivado de la acumulación de residuos urbanos en particular; así como sobre la existencia, cometido y gestión del Programa de Recogida Selectiva de Residuos Urbanos (PRU). Dicho programa consiste, en la actualidad, en la selección doméstica de dos tipos de residuos, papel-cartón y vidrio, para su posterior depósito en contenedores específicos.

En lo referente a esta cuestión, las tablas 3 y 4 nos permiten deducir que, a pesar de que aproximadamente el $50 \%$ de los encuestados afirma conocer los problemas medioambientales derivados de la basura doméstica, éstos son percibidos como de importancia menor en relación a otros problemas medioambientales: contaminación de ríos, lagos y arroyos, centrales nucleares, contaminación atmosférica y desertización. Además, para más del $50 \%$ de los individuos analizados, la problemática medioambiental se percibe tan importante como cualquier otro problema social.

En cuanto al nivel de conocimiento-percepción manifestado sobre el PRU, Tabla 5, cabe destacar el hecho de que todos los encuestados conocen la existencia de los contenedores de papel-cartón y vidrio, así como sus respectivos colores (aproximadamente el $98 \%$ en todos los casos). Sin embargo, la disponibilidad de contenedores no siempre es asociada por los individuos al reciclado de residuos urbanos, dado que solamente un $45,7 \%$ de los encuestados tiene la percepción de que una parte de la basura es reciclada.

Los resultados presentados en la Tabla 6 apuntan en la misma dirección, dado que un elevado porcentaje de los encuestados no confía en que toda la fracción depositada en los contenedores específicos sea efectivamente reciclada. En efecto, parece existir cierta desinformación acerca del cometido del PRU. Por otra parte, de dicha tabla también se deduce que existe una percepción negativa sobre la gestión del PRU llevada a cabo por el ayuntamiento de la ciudad tomada como ejemplo.

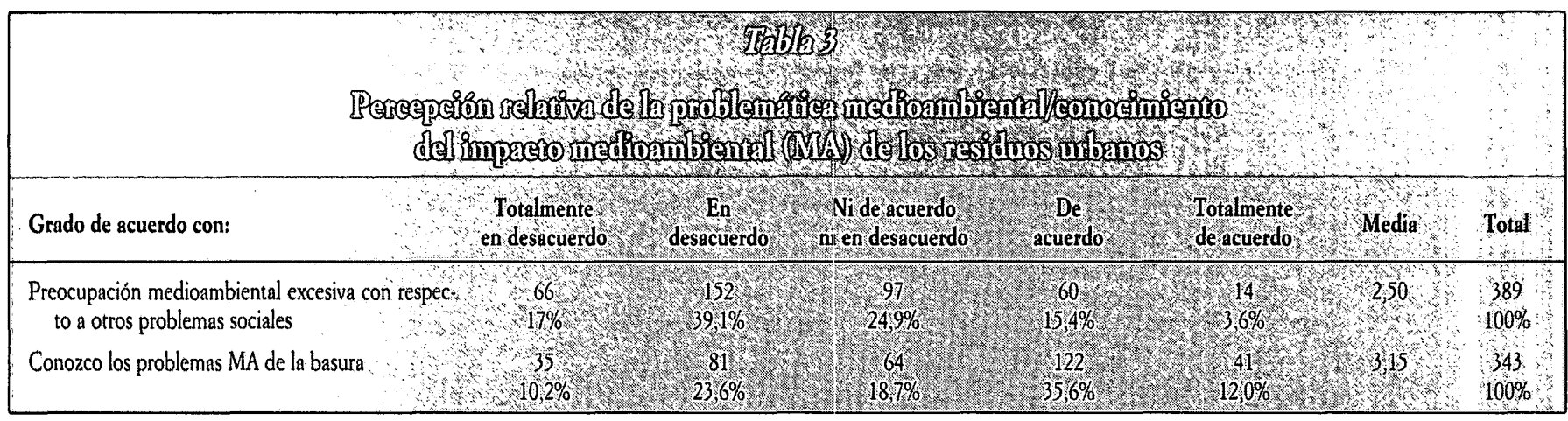

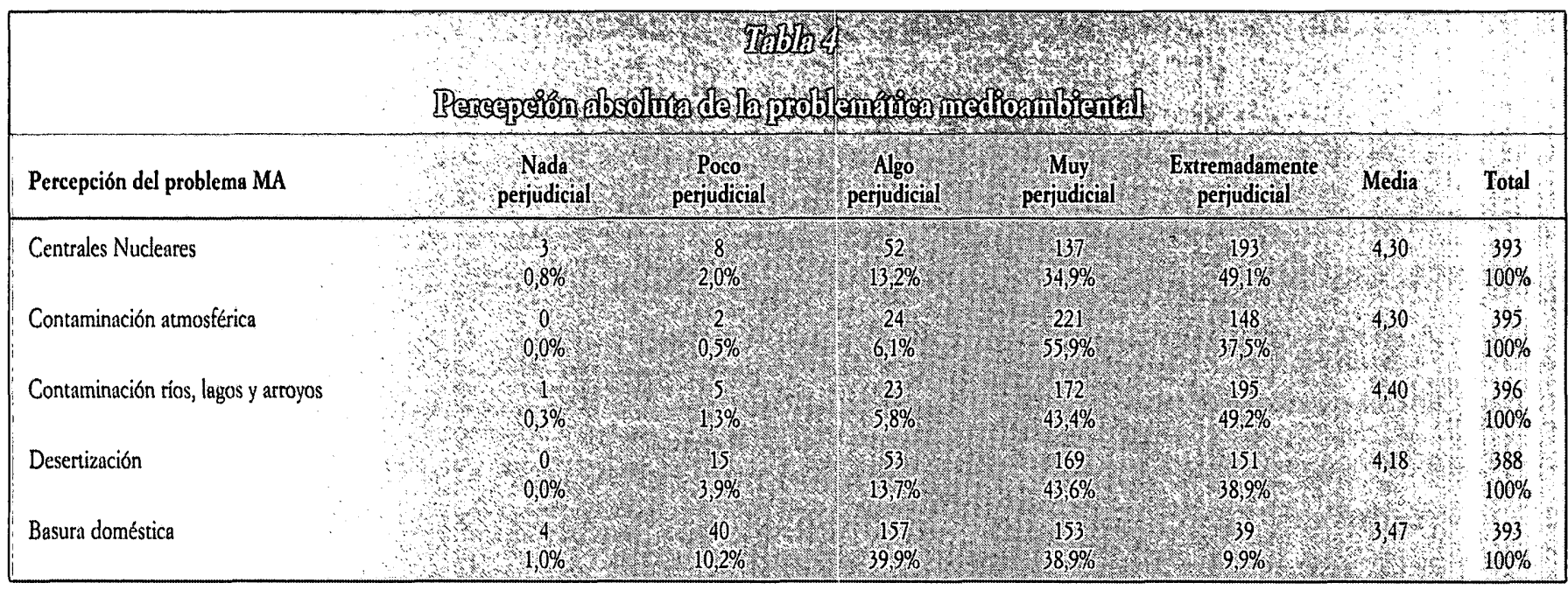




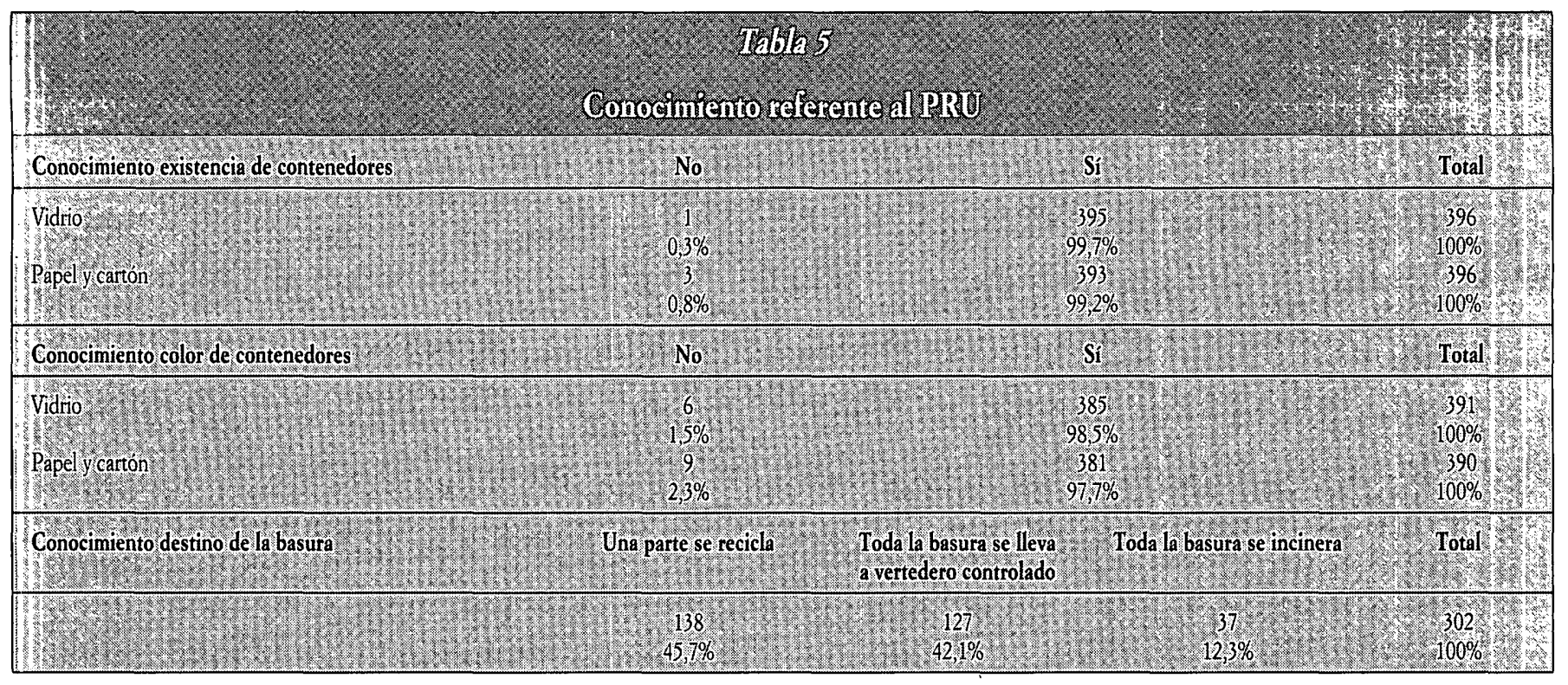

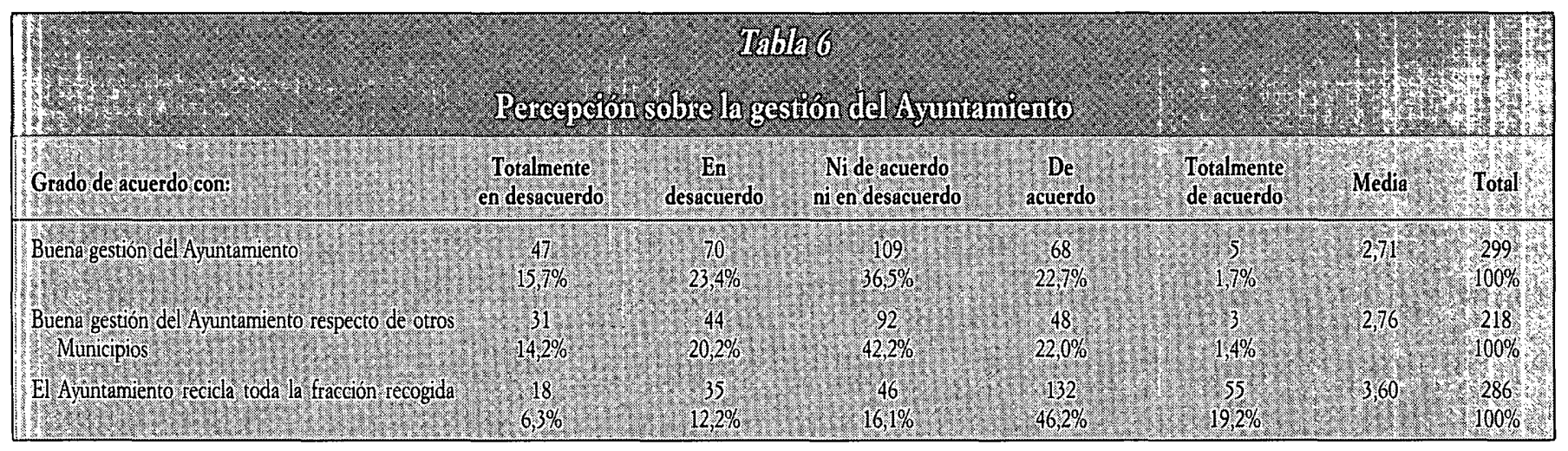

Los valores mostrados en las anteriores tablas reflejan el hecho de que los individuos analizados poseen información tanto del problema medioambiental derivado de los residuos urbanos como de la existencia de un PRU en su ciudad, aunque no conocen bien su cometido, no conceden demasiada importancia al problema medioambiental que se pretende resolver y/o no perciben una buena gestión por parte de su Ayuntamiento. En cualquier caso, y dado que el nivel de conocimiento debería influir, positivamente, sobre la actitud de un individuo hacia la participación en los PRU, los resultados parecen apuntar hacia la necesidad de una mayor información acerca de los objetivos del PRU y de la relevancia del problema medioambiental que trata de resolver. En este sentido, Mier-Terán (1994) indican que los consumidores con conciencia ecológica y/o los que están dispuestos a cambiar sus hábitos para contribuir a las soluciones medioambientales, necesitan estar informados de las consecuencias ecológicas de sus hábitos de comportamiento, lo cual dependerá del grado de transparencia del mercado y la claridad en la percepción de las consecuencias de dichos comportamientos.

La segunda cuestión de interés para el análisis radica en el grado de responsabilidad ciudadana que perciben los encuestados a la hora de resolver los problemas medioambientales en general, y el problema de la acumulación de residuos urbanos en particular. En este sentido, la Tabla 7 muestra una percepción generalizada de que, desde el punto de vista individual, no resulta difícil dedicar cierto esfuerzo personal para la resolución de los problemas medioambientales. De hecho, una buena parte de los encuestados percibe una participación generalizada de los ciudadanos en el PRU.

Por lo tanto, cabe constatar la actitud positiva de la mayor parte de la muestra analizada hacia el problema de nuestro interés. Sin embargo, la percepción de responsabilidad no parece traducirse en una actitud positiva hacia las posibles soluciones políticas que pueden implementar las autoridades públicas para la resolución de los problemas medioambientales. La Tabla 8 refleja 


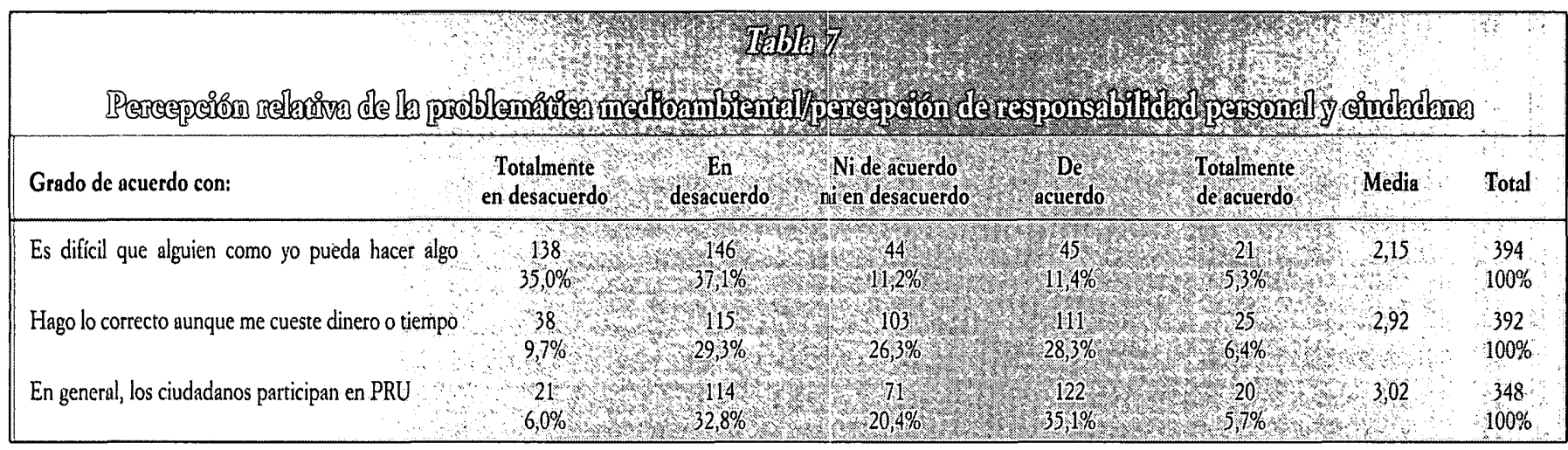

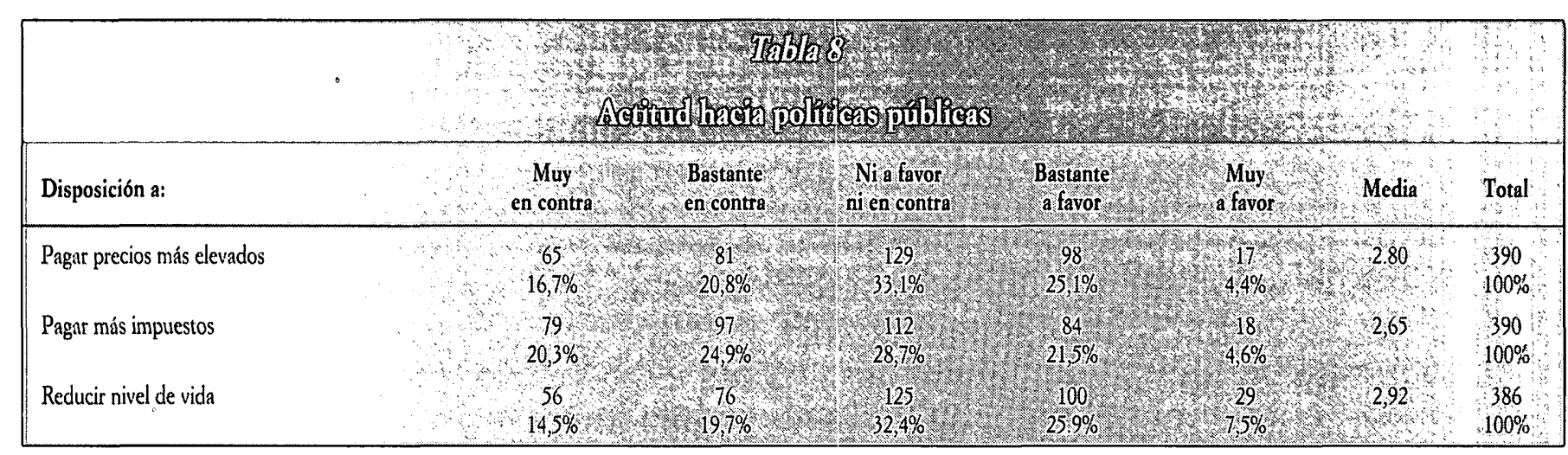

las actitudes de los encuestados respecto de dos grandes tipos de instrumentos públicos a través de los cuales los ciudadanos pueden contribuir en la protección del entorno natural: precios e impuestos. El empleo de ambos está orientado, en principio, a la internalización de los costes medioambientales. Así, en el caso que nos ocupa, la gestión de los residuos urbanos, es tradicional el uso de tasas de vertido, cuya aplicación permite recuperar el coste del servicio. De hecho, tal tipo de tasas está enriqueciendo su estructura en algunos países de la Unión Europea ${ }^{8}$, para incentivar algunos modos de gestión de los residuos urbanos.

En lo que se refiere a los precios, el empleo de tal instrumento pretende modificar la conducta de los sujetos, dándose por supuesto que la demanda es elástica respecto de los precios. En general, la vía para canalizar la internalización de costes medioambientales responde a la introducción de impuestos ecológicos. A este objetivo responde, por ejemplo, la Ley de los ecoimpuestos que introduce Bélgica en 1993, gravando una serie de productos, como los envases de bebidas, productos desechables, embalajes industriales o el papel. De esta manera, se intenta presionar tanto a productores como a consumidores para que adopten el comportamiento alternativo que ofrecen las exenciones que se establecen a estos ecoimpuestos.

El examen de la citada Tabla 8 permite comprobar que existe un cierto rechazo social a pagar precios más elevados o impuestos adicionales, evidencia ésta del todo razonable. Con todo, conviene subrayar que el rechazo a los precios es superior al correspondiente a los impuestos. Las limitaciones de la muestra impiden dar rango de generalidad a tal conclusión; en todo caso, este resultado incita a conjeturar que la vía impositiva puede ser un instrumento de internalización y financiación de los costes medioambientales más consistente con las preferencias sociales. Ello es especialmente importante si se tiene en cuenta, además, que la elasticidad-precio de sustitución entre productos con envases diferentes suele ser relativamente reducida.

La aptitud, favorable o desfavorable, hacia la protección del medio ambiente, constituye sin duda un factor determinante de la participación ciudadana en cualquier tipo de iniciativa medioambiental. Sin embargo, en lo que respecta al PRU analizado, no resulta menos relevante la percepción de un conjunto de dificultades inherentes a la participación. Siendo ésta la siguiente cuestión de interés para el análisis, ha sido considerada la percepción de dos tipos de dificultades: las dificultades personales, relacionadas en concreto con el tiempo y el espacio disponibles"para el individuo; y las relacionadas con la infraestructura del sistema de recogida, como la distancia hasta el punto de depósito, la incomodidad inherente al depósito y las condiciones en las que se encuentra el contenedor. 


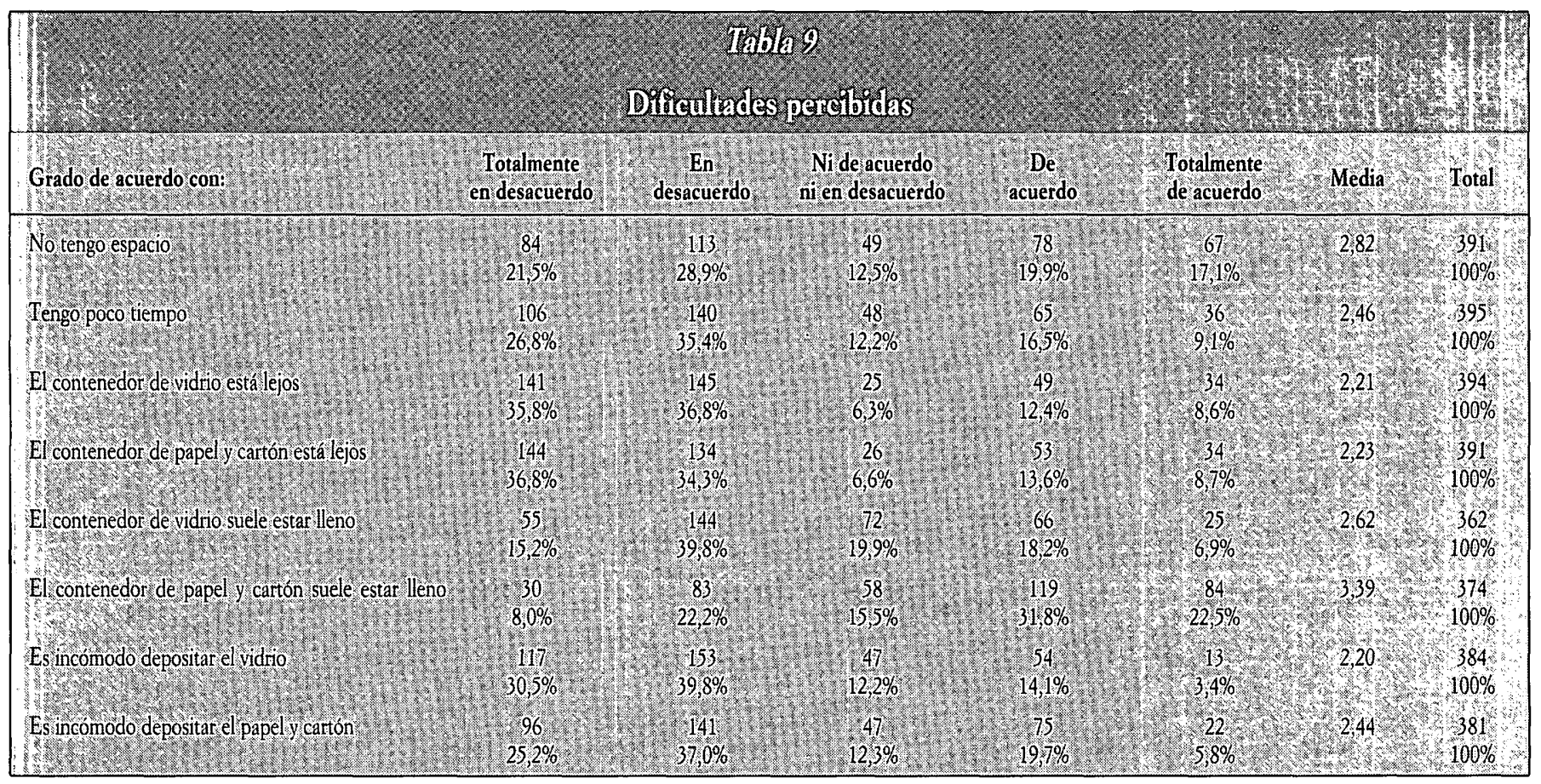

Los resultados obtenidos, reflejados en la Tabla 9, muestran que, en relación a su situación personal, el $37 \%$ de los encuestados manifiesta no tener espacio en su domicilio para almacenar los residuos urbanos, mientras que el $26 \%$ manifiesta no disponer del tiempo necesario. En cuanto a las condiciones inherentes al sistema de depósito y recogida, el $21 \%$ percibe lejanía entre el contenedor y su domicilio, el $20 \%$ percibe incomodidades en el sistema de depósito de los residuos y, en particular, respecto a los contenedores de papel y cartón, se observa un sesgo hacia el acuerdo con el hecho de que los contenedores suelen estar llenos. Estas dificultades percibidas deberían disminuir la capacidad/habilidad del ciudadano para participar en el PRU, por lo que existen implicaciones directas en el com- portamiento del individuo que deberían ser consideradas por las autoridades competentes. En concreto, deberían ser atendidas las cuestiones relacionadas con el número de contenedores disponibles en la ciudad, con el diseño de dichos contenedores y con la frecuencia de recogida de los residuos depositados.

Sin embargo, a pesar de las dificultades percibidas, los datos muestran que la mayor parte de los individuos encuestados participan en el Programa analizado, situándose el nivel de participación en cifras próximas al 79\% (Tabla 10). Se aprecia también que la participación en los dos tipos de recogida selectiva es similar.

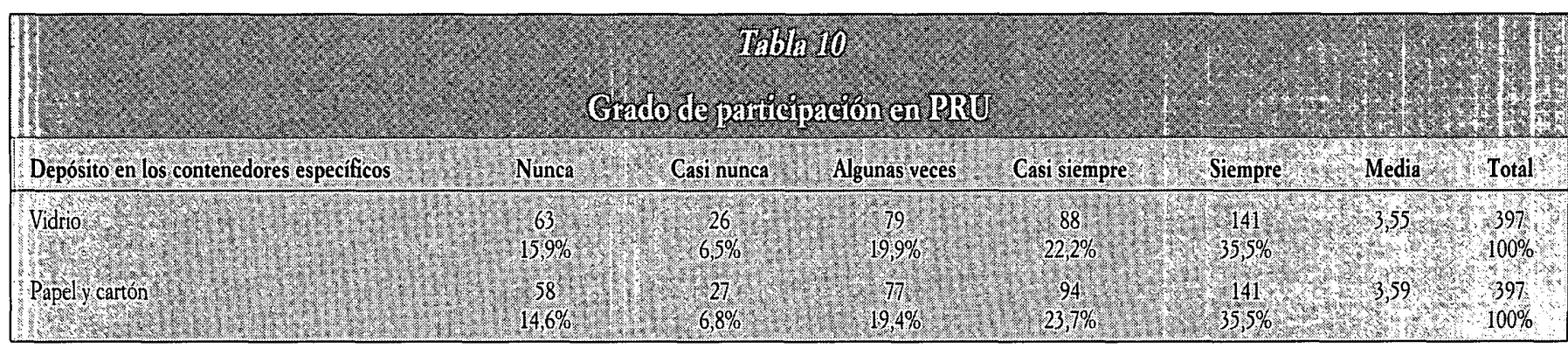

El análisis de la forma de participación, Tabla 11, indica que la unidad familiar está implicada desde hace más de dos años y que, en la mayor parte de los casos, participan todos los componentes de la unidad familiar. La frecuencia de depó- sito se realiza, en los dos tipos de residuo considerados, una vez a la semana o cada quince días. En cuanto al volumen de residuos depositado cada vez, se aprecian algunas diferencias que apuntan que el número de bolsas/cajas de cada depósito 


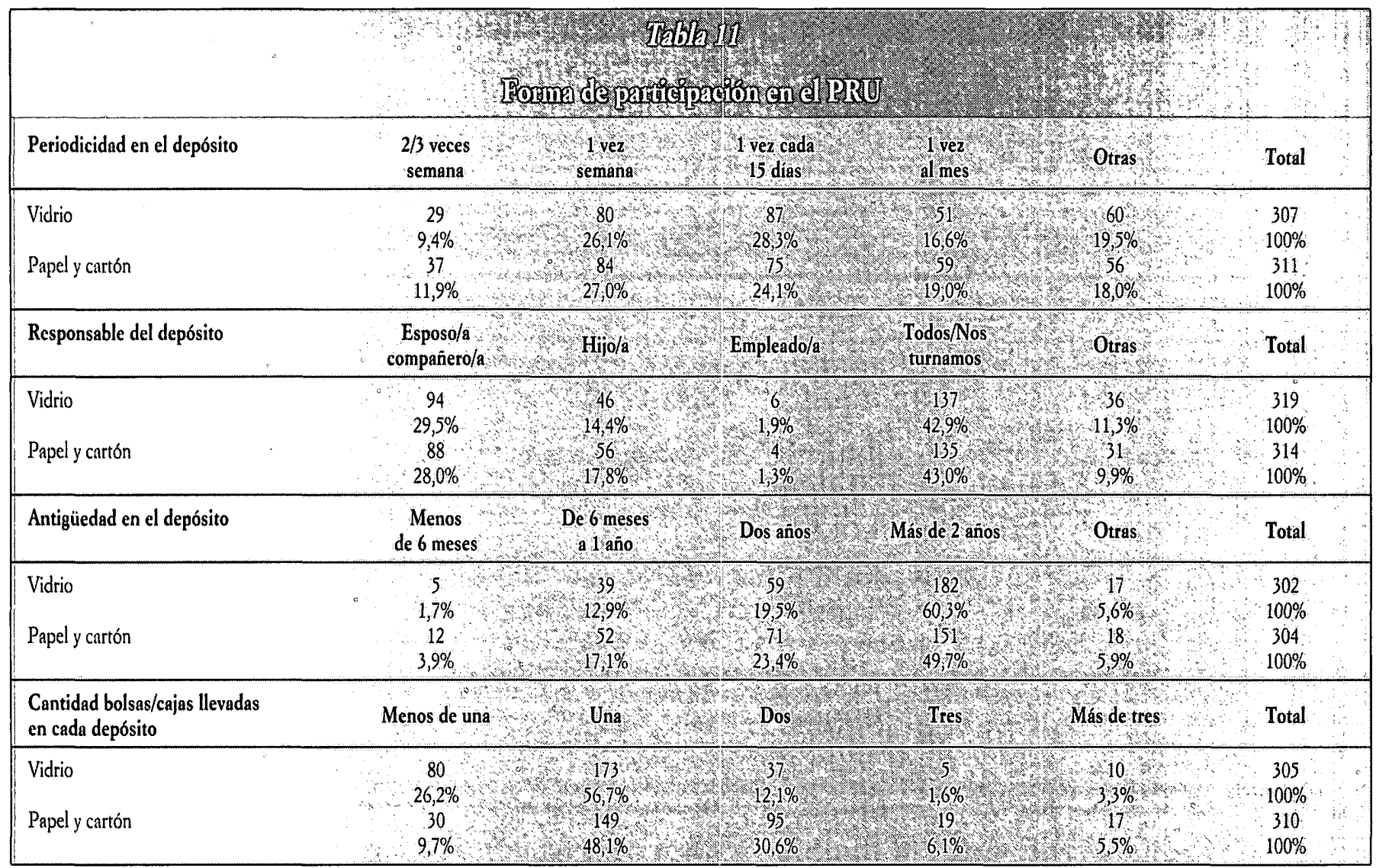

es mayor en el caso del papel-cartón que en el caso del vidrio. Este resultado puede facilitar una posible explicación al hecho de que sea el contenedor para papel-cartón el que en mayor número de ocasiones encuentra lleno el ciudadano.

El Plan de Ordenación de la Gestión de Residuos Urbanos de la Comunidad Autónoma analizada (Aragón) contempla la próxima recogida selectiva de una tercera fracción de residuos, plásticos, latas y tetra-bricks, por lo que es de nuestro interés el estudio de las intenciones manifestadas por los individuos analizados a participar en dicha solución. Cabe esperar, dadas las creencias y actitudes mostradas previamente, así como el alto nivel de participación en el actual PRU, un alto nivel de aceptación de dicho Programa.

Los datos obtenidos (Tabla 12) muestran que, efectivamente, la disposición a participar en el PRU de la tercera fracción es alta, ya que menos de un $15 \%$ de los individuos manifiesta no estar dispuesto a participar en la recogida selectiva de este tipo de residuos. La periodicidad prevista de depósito por el participante potencial es de uno o dos días, viéndose la participación beneficiada, principalmente, por el ofrecimiento de información, por parte de las autoridades competentes, sobre el destino de los residuos y por el hecho de que el depósito pudiera ser realizado en bolsas.

\section{Conclusiones}

Los psicólogos generalmente aceptan la existencia de un nexo de unión entre el nivel de conocimiento sobre la problemática medioambiental que posea un individuo y el comportamiento de defensa del entorno que posteriormente desarrolle (CORTESE, 1992; SMITH, 1992). El conocimiento se ve incrementado por la información recibida. Sin embargo, conviene señalar que la información no llega siempre, ni en su totalidad, a las personas a las que se dirige, ya que éstas atienden a una pequeña parte de la información que reciben. Además, esta información debe ser comprendida para que realmente ejerza el efecto deseado sobre el comportamiento. Así, en lo que se refiere a la defensa medioambiental, la comprensión de los temas ecológicos y de reciclado es un importante predictor del comportamiento de reciclaje, incluso más importante que la actitud medioambiental (ARBUTHNOT, 1977).

Los resultados aquí obtenidos muestran un alto nivel de conocimiento de la existencia del PRU analizado, así como de los problemas medioambientales derivados de la basura doméstica. Así, la muestra analizada considera que ello tiene la misma importancia que problemas sociales de primera magnitud. 


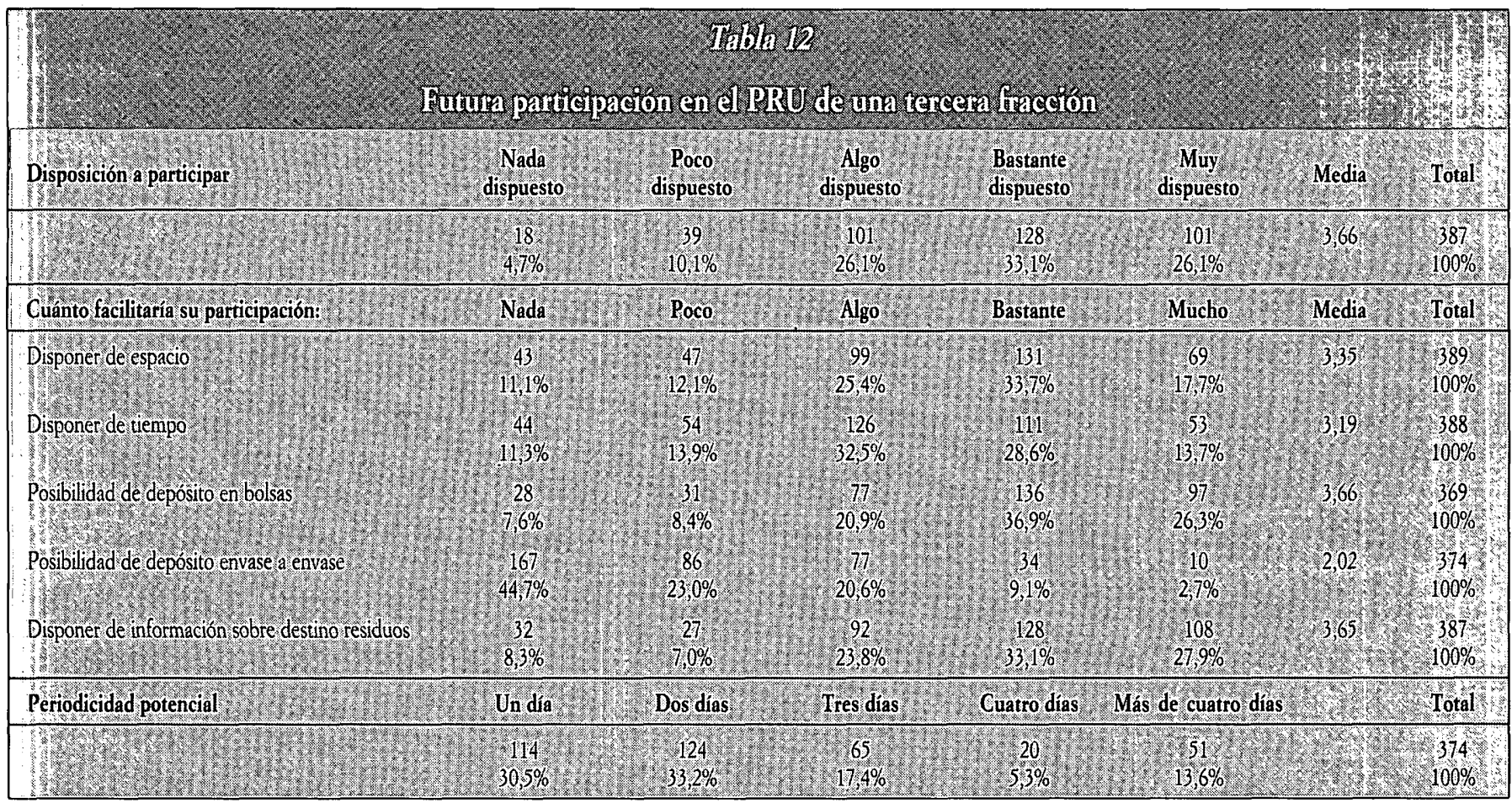

Sin embargo, existe un cierto rechazo social hacia la utilización de precios e impuestos como instrumentos de preservación del entorno. Con todo, se observa un mayor rechazo a las políticas soportadas en precios que a las desarrolladas a través de impuestos, por lo que la vía impositiva podría ser el instrumento de internalización y financiación de los costes medioambientales más consistente con las preferencias sociales.

La participación en PRU no implica, directamente, que los individuos deban soportar una serie de costes monetarios; sin embargo, sí que existen una serie de dificultades percibidas, tanto personales como del sistema de recogida utilizado, que podrían disminuir dicha participación. A pesar de emerger las dificultades personales, tiempo y espacio, como las que limitan en mayor medida la participación en los PRU, su incidencia no es la que inicialmente se esperaría. En la muestra analizada, la mayor parte de los hogares participan en el PRU de su ciudad. Igualmente, se detecta un alto nivel de futura participación en la recogida de un tercer tipo de residuos, plásticos, latas y tetra-bricks.

Los resultados aquí obtenidos deberán ser comprobados a través de la formulación de un modelo que permita establecer los factores determinantes de los comportamientos sociales relacionados con la participación en los Programas de Residuos Urbanos. Así, surge una línea de investigación que será abordada en un futuro próximo, con el objetivo de determinar si dichos factores son sensibles al signo e intensidad de algunas políticas públicas; de aquí se derivan contenidos decisionales inmediatos.
* Los autores agradecen la financiación facilitada por CSID-DGA.

** Universidad de Zaragoza.

${ }^{1}$ El primer vestigio de la normativa medioambiental en España es el Reglamento de Actividades Molestas, Insalubres, Nocivas y Peligrosas de 1961 (EsTEVAN, 1991, p. $x v)$.

${ }^{2}$ Resolución del Consejo del 1 de febrero de 1993.

3 Información facilitada por el Ministerio de Medio Ambiente (1998).

4 Datos facilitados por Ecoembalajes España, S. A., en el Seminario «Los Sistemas Integrados de Gestión de Residuos: Aplicación a los Residuos de Envases», Zaragoza, enero 1999.
5 Por ejemplo, la Directiva 91/156/CEE, relativa a los residuos, establece que la protección eficaz del medio ambiente requiere, no sólo garantizar la eliminación responsable de los residuos, sino también la reducción en origen, la recuperación y el reciclado.

${ }^{6}$ Esta Ley fija, además, unos objetivos intermedios de reciclado que deben cumplirse en el plazo de treinta y seis meses desde su entrada en vigor así como una reducción de, al menos, el $10 \%$ en peso de la totalidad de los residuos generados en un plazo de cuatro años.

${ }^{7}$ En lo que respecta a los envases domésticos, esta Ley ofrece a los envasadores dos opciones: (i) poner estos envases en el mercado a través del Sistema de 
Depósito, Devolución y Retorno; (ii) adherir estos envases a un Sistema Integrado de Gestión de Residuos de Envases (SIG). Dada la complejidad y la responsabilidad que se deriva de la primera de las opciones, todos los envases domésticos se han adherido a un SIG. Según este sistema, el envasador paga a un SIG una cierta cantidad, dependiendo del tipo de material y del peso del envase, por cada producto envasado que pone en el mercado. De esta manera, ni el envasador, ni el comerciante o distribuidor, tienen responsabilidad en la gestión del residuo de envase. La gestión le compete al SIG en colaboración con los Ayuntamientos, que son los encargados de llevar a cabo la recogida selectiva de los residuos de envases, poniendo a disposición de los ciudadanos contenedores específicos para cada tipo de material. El sobrecoste que tienen los Ayuntamientos por estas actividades, frente a lo que les costaba la recogida en masa y el depósito en vertedero, lo paga el SIG a través de acuerdos económicos fijados en los convenios de colaboración.

${ }^{8}$ Dentro de la Unión Europea, una de las primeras aplicaciones de este impuesto se observa en Dinamarca que, desde 1986, establece un impuesto a la eliminación de residuos con una tasa diferenciada más elevada para el vertido de residuos que para la incineración. La efectividad de este impuesto se manifiesta en la reducción del vertido, desde 1985 a 1993 , desde un $39 \%$ a un $26 \%$, a la vez que la reutilización y el reciclaje se han incrementado desde un $35 \%$ a un $50 \%$. Más recientemente, Holanda introduce en 1995 un impuesto sobre el vertido de residuos aunque la incineración no se grava debido a la falta de espacio. La recaudación de estos impuestos se integra en los presupuestos generales de estos países formando parte de la reforma fiscal verde que llevan a cabo.

\section{Bibliografía}

ARbutrinot, J. (1977), «The Role of Attitudinal and Personality Variables in the Prediction of Environmental Behavior and Knowledge», Environment and Bebavior, vol. 9, No. 2, pp. 217-232.

ButTel, F. H., y FLINN, W. L. (1974), «The Structure of Support for the Environmental Movement, 1968-1970», Rural Sociology, vol. 39, No. 1, pp. 56-69.

- (1978), «Social Class and Mass Environmental Beliefs: A Reconsideration», Environment and Behavior, vol. 10, No. 3, pp. 433-450.

CORTESE, A. (1992), «Education for an Environmentally Sustainable Future», Environmental Science and Technology, vol. 26, pp. 1108-1114.

Dunlap, R. E., y Catron Jr., W. R. (1979), «Environmental Sociology», Annual Review of Sociology, No. 5.

Estevan, M. T. (1991), Implicaciones Económicas de la Protección Ambiental de la CEE: Repercusiones en España, Madrid: Instituto de Estudios de Prospectiva. Ministerio de Economía y Hacienda.

FinNIE, W. C. (1973), «Field Experiment in Litter Control», Environment and Bebavior, No. 5, pp. 123-144.

Franco, J., y Huerta, E. (1996), «Determinantes de la Participación Ciudadana en Programas de Reciclaje de Residuos Sólidos Urbanos», Investigaciones Económicas, vol. XX, No. 2, pp. 271-280.

JaCobs, H. E.; Balley, J. S., y Crews, J. I. (1984), «Development and Analysis of a Community Based Resource Recovery Program», Journal of Applied Behavioral Analysis, vol. 17, pp. 127-145.
Lowe, G. D., y Pinhey, T. K. (1982), «Rural-Urban Differences in Support for Environmental Protection», Rural Sociology, vol. 47, No. 1, pp. 114-128.

McEvoy, J. (1972), «The American Concern With Environment», en W. BurCH et al. (eds.), pp. 214-236, Social Behavior, Natural Resources and the Environment, New York: Edit. Harper \& Row.

Mier-Terán, J. J. (1994), «Posibilidad de Formulación de Políticas de Marketing Ecológico», Actas del VI Encuentro de Profesores Universitarios de Marketing, San Sebastián, pp. 505-514.

Ministerio de Medio Ambiente (1998), Medio Ambiente en España 1997, Serie Memorias, Madrid: Dirección General de Calidad y Evaluación ambiental.

SMITH, G. (1992), Education and the Environment: Learning to Live With Limits, Albany: SUNY Press.

Tognacci, L. N.; Weigel, R. H.; WILden, M. F., y Vernon, D. T. A. (1972), «Environmental Quality: how Universal is Public Concern?», Environment and Behavior, vol. 4, No. 1, pp. 73-87.

Tremblay Jr., K. R., y Dunlap, R. E. (1978), «Rural-Urban Residence and Concern With Enviromental Quality: A Replication and Extension», Rural Sociology, No. 43 , pp. 474-491.

VINING, J., y EBreo, A. (1990), «What Makes a Recycler? A Comparison of Recyclers and Non-Recyclers», Environment and Bebavior, vol. 22, pp. 55-73.

Witmer, J. F., y Geller, E. S. (1976), «Facilitating Paper Recycling: Effects of Prompts, Raffles and Contests», Journal of Applied Psychology, No. 63, pp. 73-80. 


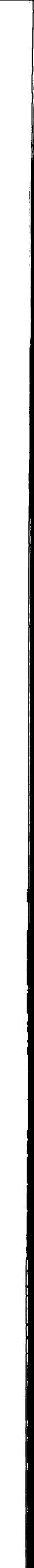

